\title{
The Coparenting Relationship Scale-Father's Prenatal Version
}

\author{
Tiago Miguel Pinto $^{1}$ - Bárbara Figueiredo ${ }^{1}$ Mark E. Feinberg ${ }^{2}$
}

(c) Springer Science+Business Media, LLC, part of Springer Nature 2018

\begin{abstract}
This study aimed to examine the psychometric characteristics of the Coparenting Relationship Scale when administered in fathers during pregnancy. During the first trimester of a partner's pregnancy, 91 primiparous fathers completed the Coparenting Relationship Scale-Father's Prenatal Version (CRS-FPV), and self-report measures of depressive and anxious symptoms, adult attachment, and partner's relationship quality. The CRS-FPV revealed good internal consistency. Exploratory factor analysis revealed four factors: lack of coparenting support, coparenting conflict, coparenting disagreement, and coparenting undermining. Confirmatory factor analysis revealed a good model fit. Significant associations between the CRSFPV and the original CRS subscales were found. Hypothesized associations between the CRS-FPV subscales and individual (depressive and anxious symptoms and adult attachment) and dyadic (partner's relationship quality) constructs were also significant. The present study suggested that the CRS-FPV is a reliable multidimensional measure to assess coparenting in fathers during pregnancy.
\end{abstract}

Keywords Coparenting Relationship Scale $\cdot$ Psychometric characteristics $\cdot$ Pregnancy $\cdot$ Fathers $\cdot$ Depressive and anxious symptoms · Adult attachment · Partner's relationship quality

\section{Introduction}

According to Feinberg (2003), coparenting is defined as the involvement of both parents in education, responsibilities, and decisions about their children's lives, focusing on interparental interactions associated with adults' functions and expectations about their role as parents. Constructing a coparenting relationship is an important developmental task during the transition to parenthood (e.g., Altenburger et al. 2014; Favez et al. 2013; Van Egeren 2004). This relationship is built progressively across the transition to parenthood and is already operative during pregnancy (e.g., Altenburger et al. 2014; Favez et al. 2013; Van Egeren and Hawkins 2004). For example, during pregnancy parents start to develop mental representations of themselves as coparents (e.g., Feinberg 2003; Van Egeren 2003). From this perspective, when partners start to discuss issues related

Tiago Miguel Pinto

tmpinto@psi.uminho.pt

1 School of Psychology, University of Minho, Campus of Gualtar, 4710-057 Braga, Portugal

2 Prevention Research Center, Pennsylvania State University, Park University, Parkville, MO, USA to coparenting (e.g., how they will divide caregiving) they have already begun the process of coparenting, long before childbirth (e.g., Altenburger et al. 2014; Favez et al. 2013; Van Egeren and Hawkins 2004).

The way that both fathers and mothers adjust to their new roles as coparents is determined and influenced by multiple factors. Feinberg's ecological model (2003) suggests that coparenting is shaped by four main groups of factors: individual parental characteristics, child characteristics, interparental relationship and stress and support contextual sources. Studies suggested that gender affects coparenting behavior (e.g., Schoppe-Sullivan et al. 2008). During pregnancy, constructing a coparenting relationship may be especially important for fathers. While mothers undergo more physical changes of pregnancy, fathers seem to have more leeway in the degree to which they are forward-looking and preparing to become a parent and a coparent (e.g., Schoppe-Sullivan et al. 2014). Father's depressive and anxious symptoms were found to be negatively associated with coparenting support and positively associated with coparenting conflict (e.g., Bronte-Tinkew et al. 2007, 2009, 2010). Studies also showed negative associations between the secure dimension of father's adult attachment and coparenting conflict (e.g., Talbot et al. 2009). Other studies found that lower couple 
relationship quality is associated with lower coparenting support and higher coparenting conflict (e.g., Bronte-Tinkew et al. 2009). Namely, prenatal couple relationship quality were found to predict coparenting behavior at 3-month postpartum (e.g., McHale et al. 2004).

Feinberg (2003) proposed an empirically based ecological model, conceptualizing coparenting in four interrelated dimensions: childrearing agreement, the division of labor, support (vs. undermining), and the joint family management. To assess the dimensions of coparenting, Feinberg et al. (2012) developed a multidimensional self-report measure of coparenting - the Coparenting Relationship Scale (CRS). This measure assesses coparenting quality with several subscales representing each dimension of coparenting, allowing one to assess more precisely the relation between these different dimensions and parents' adjustment (e.g., Solmeyer and Feinberg 2011), partner's relationship quality (e.g., Bronte-Tinkew et al. 2009), parenting (e.g., Pedro et al. 2012), and child outcomes (e.g., Scrimgeour et al. 2013).

Recently, some studies have analyzed the development of coparenting during pregnancy, using observational measures (e.g., Altenburger et al. 2014; Favez et al. 2013). These measures contribute to the study of coparenting interactions during this period. However, when compared with observational measures self-report measures present several advantages. A self-report measure may allow the assessment of coparenting mental representations during pregnancy and may possibly be more accurate to detect which parents are at risk of coparenting maladjustment. This study aimed to examine the psychometric characteristics of the Coparenting Relationship Scale when administered in fathers during pregnancy. We administered the measure to expectant fathers to assess with exploratory factor analysis whether the original subscale structure would remain intact or require alteration for fathers during pregnancy. We termed the new scaling structure for the pregnancy version of the measure the Coparenting Relationship Scale-Father's Prenatal Version (CRS-FPV).

\section{Method}

\section{Participants}

The sample was composed of 91 primiparous fathers recruited at the first trimester of pregnancy in three Health Services in Portugal. Exclusion criteria were as follows: not able to read or write Portuguese, living in Portugal in time $<10$ years, multiparous fathers, multiple gestations, and pregnancies with gestational problems. From the 130 participants contacted, $93.1 \%(N=121)$ agreed to participate and signed an online informed consent form, and 70\% $(N=91)$ completed the Coparenting Relationship Scale-Father's Prenatal Version.

Nearly, all the participants were Portuguese (96.7\%), Caucasian (87.9\%), and Catholic (98.9\%). More than a half of the participants were aged between 30 and 39 years old $(M=31.25, \mathrm{SD}=4.52)$; were from a high or medium socio-economic level (59\%); and were employed in manual (qualified or not qualified) professions, for more than 7 years $(55.3 \%)$. The majority of the participants were married or cohabiting $(92.3 \%)$, and living with the partner without any other family members in the household (76.9\%; see Table 1$)$.

\section{Procedure}

We conducted the present research in accordance with the Helsinki Declaration and received previous approval from the Ethical Commissions of all institutions involved. Participants were randomly recruited (October 2013-April 2014) in the obstetrics outpatient service waiting room after the first fetal ultrasound (8-14 gestational' weeks). The aims and the procedures of the study were explained. Participants completed the CRS-FPV and self-report measures of

Table 1 Fathers' socio-demographic characteristics

\begin{tabular}{|c|c|}
\hline Characteristic & $N=91(\%)$ \\
\hline \multicolumn{2}{|l|}{ Age (years) } \\
\hline $20-29$ & 35.2 \\
\hline $30-39$ & 61.5 \\
\hline \multicolumn{2}{|l|}{ Socio-economic level } \\
\hline High & 33.3 \\
\hline Medium high & 14.1 \\
\hline Medium & 25.7 \\
\hline Medium low & 19.2 \\
\hline Low & 7.7 \\
\hline \multicolumn{2}{|l|}{ Professional status } \\
\hline Employed & 89.0 \\
\hline Unemployed & 8.8 \\
\hline Household or student & 2.2 \\
\hline \multicolumn{2}{|l|}{ Education (in years) } \\
\hline$<9$ & 9.9 \\
\hline $9-12$ & 52.7 \\
\hline$>12$ & 37.4 \\
\hline \multicolumn{2}{|l|}{ Matrimonial status } \\
\hline Married & 63.7 \\
\hline Cohabitation & 28.6 \\
\hline Single & 7.7 \\
\hline \multicolumn{2}{|l|}{ Household } \\
\hline Partner & 76.9 \\
\hline Partner and family & 13.2 \\
\hline Family (only) & 7.7 \\
\hline
\end{tabular}


depressive and anxiety symptoms, adult attachment, and partner's relationship quality.

\section{Measures}

\section{Coparenting Relationship Scale}

The Coparenting Relationship Scale (CRS; Feinberg et al. 2012) is a 35-item self-report scale scored on a 7-point Likert scale designed to assess the four domains of coparenting proposed by Feinberg (2003). This measure is comprised by seven subscales: (1) the Coparenting agreement subscale (four items) that assesses the degree that each parent agrees with matters related to the child's education; (2) the Coparenting closeness subscale (five items) that assesses the degree to which coparenting enhanced intimacy and strengthened the partner's relationship; (3) the Exposure to conflict subscale (five items) that assesses the degree that parents expose the child to conflicts related to their education; (4) the Coparenting support subscale (six items) that assesses the perception of coparenting support from the partner; (5) the Coparenting undermining subscale (six items) that assesses the perception that the coparental relationship is regulated by critics, guilt and competition between the parents; (6) the Endorse partner parenting subscale (seven items) that assesses one's own positive attitude toward the partner's parenting; and (7) the Division of labor subscale (two items) that assesses how the parents share and coordinate the responsibilities of child care. This measure showed good internal consistency in fathers (Cronbach's $\alpha=.93$ for total scale; .93 for coparenting agreement subscale; .72 for coparenting closeness subscale; .88 for exposure to conflict subscale; .89 for coparenting support subscale; .82 for coparenting undermining subscale; .72 for endorse partner parenting subscale; and $\mathrm{r}(147)=.42$ for division of labor subscale).

The CRS-FPV consists in a prenatal adaptation of the CRS for fathers. The CRS-FPV is based on Portuguese spoken and written in Portugal. To compose the CRS-FPV, all CRS items were translated to Portuguese, back-translated to English and adapted for prenatal period.

\section{CRS-FPV-Related Constructs}

We used four measures to assess CRS-FPV criterion validity-individual (depressive and anxious symptoms and adult attachment) and dyadic (partner's relationship quality) constructs. We used the Edinburgh Postnatal Depression Scale (EPDS; Cox et al. 1987) to assess depressive symptoms. EPDS is a 10-item self-report scale scored on a 4-point Likert scale, designed to assess the intensity of depressive symptoms within the previous 7 days. This measure has been used in several studies with fathers during pregnancy (e.g.,
Figueiredo and Conde 2011; Matthey et al. 2001; Parfitt and Ayers 2014). EPDS Portuguese version showed good internal consistency during pregnancy $(\alpha=.88$; Figueiredo and Conde 2011). In the present sample, coefficient $\alpha$ was .80 .

The State-Trait Anxiety Inventory (Spielberger et al. 1983 ) is a self-report scale composed of two subscales, the state anxiety and the trait anxiety, each containing 20 items scored on a 4-point Likert scale. We used the State Anxiety Inventory (STAI-S) that measures the temporary condition of "state anxiety" (anxiety in a specific situation), to assess anxious symptoms. Several studies have been used this measure during pregnancy with fathers (e.g., Figueiredo and Conde 2011; Figueiredo et al. 2008). STAI-S Portuguese version showed good internal consistence $(\alpha=.88$; Biaggio et al. 1976). In the present sample, coefficient $\alpha$ was .87.

We used the Experiences in Close Relationship Scale (ECR; Brennan et al. 1998) to assess adult attachment. The ECR is a 36-item self-report scale scored on a 7-point Likert scale and is composed of two subscales designed to assess the avoidance (18 items) and anxiety (18 items) dimensions of adult attachment. Higher scores on the avoidance and anxiety subscales indicate higher levels of attachment anxiety and attachment avoidance, respectively. ECR Portuguese version showed good internal consistency $(\alpha=.86$ for the total scale; .88 for the avoidance subscale; and .86 for the anxiety subscale; Paiva and Figueiredo 2010). In the present sample, coefficient $\alpha$ was .81 for the avoidance subscale and .85 for the anxiety subscale.

We used the Relationship Questionnaire (RQ; Figueiredo et al. 2008) to assess partner's relationship quality. The RQ is a 12-item self-report scale scored on a 4-point Likert scale. It is composed of two subscales designed to assess both positive and negative aspects of partner relationship: (1) positive relationship subscale (eight items), including a sense of support and care, affection, closeness, and joint interests and activities; and (2) negative relationship subscale (four items), including anxiety, irritability, and criticisms that have been associated with undesirable outcomes. A higher score on the positive (negative) relationship subscale means that there is a stronger dimension of positivity (negativity) in the relationship. The questionnaire showed good internal consistency $(\alpha=.79$ for the total scale; .90 for the positive subscale; and .72 for the negative subscale) and test-retest reliability ( $r=50.74$ for the total scale). In the present sample, coefficient $\alpha$ was .71 for the positive subscale and .53 for the negative subscale.

\section{Analytical Strategy}

In order to examine the psychometric characteristics of the CRS-FPV, we performed an analysis of (1) internal consistency, (2) factor structure, and (3) criterion validity. To examine (1) CRS-FPV (total scale and subscales) internal 
consistency, we examined Cronbach's alpha coefficient, item-total correlation, and mean item correlation. Good internal consistency is indicated that when the Cronbach's alpha is higher than .70, the item-total correlation is over .30, and the mean item correlation is higher than .15 (e.g., Field 2005). To examine (2) CRS-FPV factor structure, we performed an exploratory factor analysis (principal axis analysis with orthogonal rotation - varimax) and a confirmatory factor analysis (maximum likelihood estimation). We removed coefficients below .40. We computed the Chi-square statistic, the Comparative Fit Index (CFI), and the Root Mean Square Error of Approximation (RMSEA) to analyze model goodness of fit. Kline (2005) referred that a good model fit is evidenced by a non-significant Chi-square, a CFI of at least .95 , and a RMSEA of .05 or less. Additionally, we examined the associations among CRS-FPV scales, and compared the 30-item CRS-FPV with the original 35-item CRS with seven subscales (the same factor structure proposed by Feinberg et al. 2012). To examine (3) CRS-FPV criterion validity, we examined associations among the CRS-FPV subscales and individual (depressive and anxious symptoms and adult attachment) and dyadic (partner's relationship quality) constructs. We performed the statistical analyses using SPSS and SPSS Amos version 22.0 (SPSS Inc., USA).

\section{Results}

\section{Internal Consistency}

From the 35 items, we removed five items. These items reduced the CRS-FPV internal consistency $(\alpha=.58)$ and presented an item-total correlation less than .30 . The resulting overall CRS-FPV (30 items) demonstrated good internal consistency $(\alpha=.85)$. All items presented an item-total correlation above .30 (see Table 2).

\section{Factor Structure}

The Bartlett's test of sphericity result suggested that the data was adequate for principal component analysis, $\chi^{2}(435)=1999.75, p<.001$. The Kaiser-Meyer-Olkin test result for measuring sample adequacy was good $(\mathrm{KMO}=.80$; e.g., Field 2005). Four factors were generated with eigenvalues over 1 , accounting for $60.7 \%$ of the total variance: the first factor (labeled "lack of coparenting support") contained loadings for 15 items (e.g., My partner make me feel like I will be the best possible parent for our child) and contributed $23.0 \%$ of the total variance; the second factor (labeled "coparenting conflict") contained loadings for five items (e.g., Do you find yourself in a mildly tense or sarcastic interchange with your partner?) and contributed $12.9 \%$ of the total variance; the third factor (labeled "coparenting disagreement") contained loadings for six items (e.g., My partner and I will have the same goals for our child) and contributed $12.6 \%$ of the total variance; and the fourth factor (labeled "coparenting undermining") contained loadings for four items (e.g., My partner will not trust my abilities as a parent) and contributed $12.2 \%$ of the total variance (see Table 2). The placement of the "elbow" in the figure-related Cattell's scree test (1966) supported the decision to retain four factors.

Confirmatory factor analysis revealed a good model fit, $\chi^{2}(2)=2.145, p=.342$, RMSEA $=.03, \mathrm{CFI}=.99$. All CRSFPV subscales showed high loadings on the CRS-FPV latent factor. The lowest factor loading was for coparenting undermining (.68; see Fig. 1).

\section{CRS-FPV Subscales: Internal Consistency, Intercorrelations, and Correlations with Original Scale}

The Cronbach's alpha for all CRS-FPV subscales based on the four factors (created by averaging item responses in each factor) yielded values over .70 in nearly all the subscales: lack of coparenting support $(\alpha=.94)$, coparenting conflict ( $\alpha=.90)$, coparenting disagreement $(\alpha=.78)$, and coparenting undermining ( $\alpha=.69$; see Table 2$)$.

Pearson correlations revealed that the four subscales were positively correlated with the CRS-FPV (total scale) and between them (see Table 3). Likewise, Pearson correlations revealed significant correlations between the CRS-FPV with 30 and four subscales and the original CRS with 35 items and seven subscales (see Table 4).

\section{Criterion Validity}

Results revealed significant correlations between the CRSFPV subscales and individual (depressive and anxious symptoms and adult attachment) and dyadic (partner's relationship quality) constructs (see Table 5).

\section{Discussion}

The present study examined the factor structure of the CRS administered to fathers, created a revised measure for fathers (CRS-FPV), and examined reliability and validity of the new measure and subscales. Results suggested that the CRS-FPV is a reliable multidimensional self-report measure of coparenting in fathers during pregnancy. The extracted factor structure suggested four factors: lack of coparenting support, coparenting conflict, coparenting disagreement, and coparenting undermining. Confirmatory factor analysis revealed a good model fit. This CRSFPV factor structure was different than the CRS structure 
Table 2 Principal axis factoring (varimax rotation) of the CRS-FPV: factor loadings, communalities, mean item correlation, item-total correlation, and alpha if item deleted

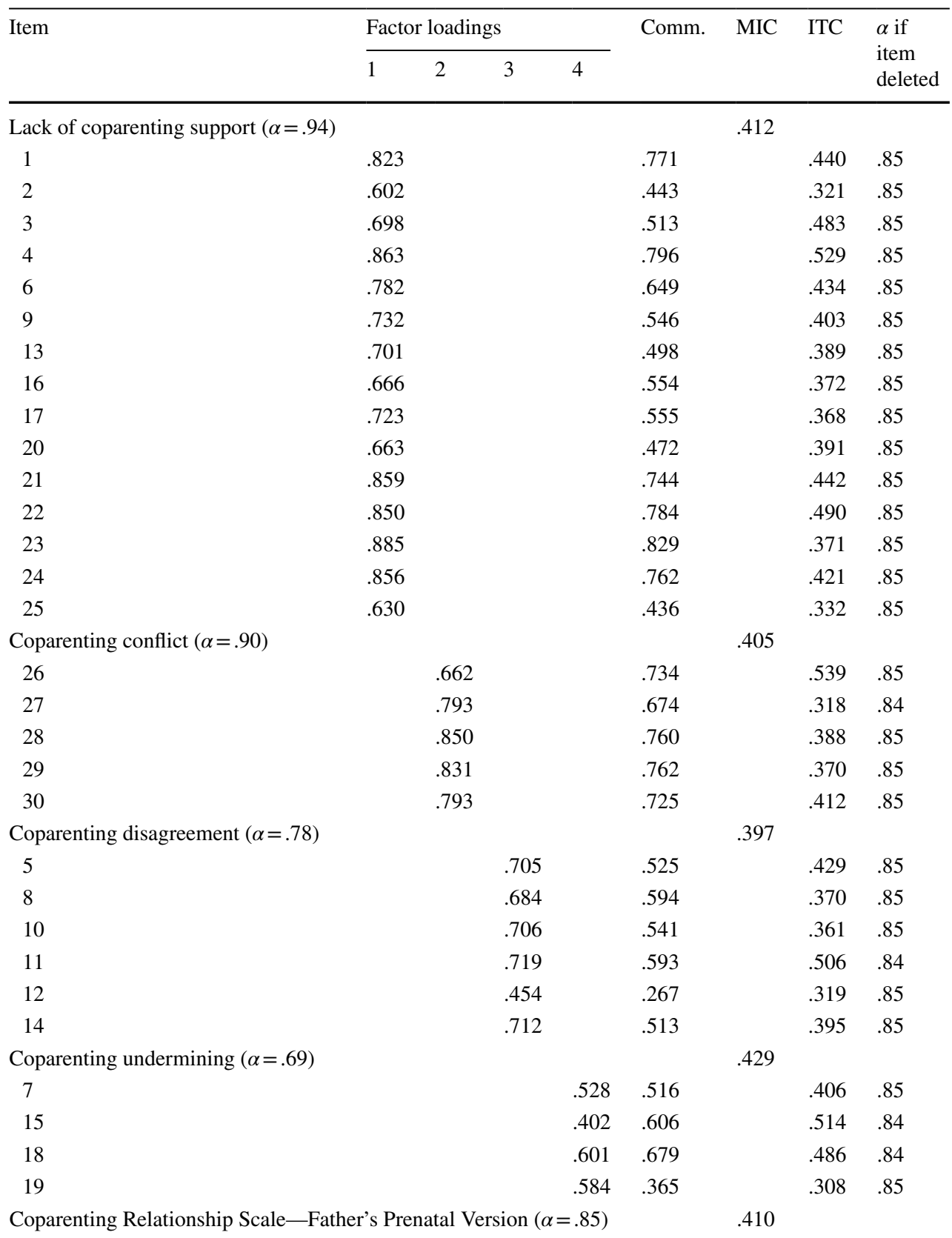

Comm. communalities, MIC mean item correlation, ITC item-total correlation

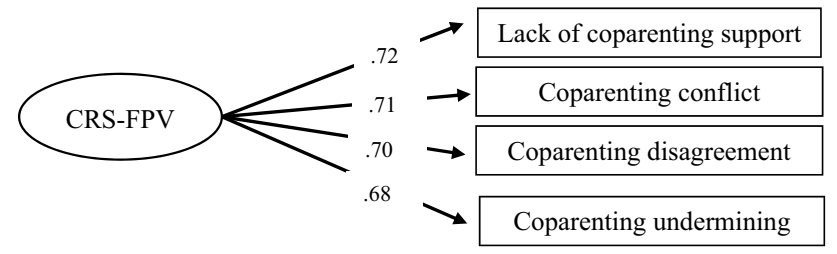

Fig. 1 Confirmatory factor analysis of the Coparenting Relationship Scale-Father's Prenatal Version and did not identify the following dimensions proposed by Feinberg et al. (2012): coparenting closeness, endorse partner parenting, and division of labor. Another difference consists in the fact that the CRS-FPV is comprised of 30 items. Conceptual similarities included the dimensions of support, conflict and undermining identified in both, the CRS and in the CRS-FPV, as important dimensions of coparenting. Differences may be explained by the fact that the CRS-FPV was developed as a measure of coparenting for fathers only, and specifically for the prenatal period before some issues (such as division of labor, endorsement 
Table 3 CRS-FPV intercorrelations

\begin{tabular}{llllll}
\hline & CRS-FPV & LCS & CC & CD & CU \\
\hline CRS-FPV & 1.00 & & & & \\
LCS & $.706^{* * *}$ & 1.00 & & & \\
CC & $.474 * * *$ & $.345^{* * *}$ & 1.00 & & \\
CD & $.696^{* * *}$ & $.311^{* * *}$ & $.495^{* * *}$ & 1.00 & \\
CU & $.618^{* * *}$ & $.311^{* * *}$ & $.480^{* * *}$ & $.486^{* * *}$ & 1.00 \\
\hline
\end{tabular}

$C R S-F P V$ Coparenting Relationship Scale-Father's Prenatal Version, $L C S$ lack of coparenting support, $C C$ coparenting conflict, $C D$ coparenting disagreement, $C U$ coparenting undermining

$* * * p<.001$

Table 4 Correlations between the 30-item CRS-FPV with four subscales and the 35 -item CRS with seven subscales

\begin{tabular}{llllll}
\hline & CRS-FPV & LCS & CC & CD & CU \\
\hline CRS & $.622^{* * *}$ & $-.805^{* * *}$ & .180 & -.070 & $.298^{* *}$ \\
CA & $-.446 * * *$ & -.205 & $-.433^{* * *}$ & $-.831^{* * *}$ & $-.356^{* *}$ \\
CCL & $.282^{* *}$ & $-.826^{* * *}$ & $-.332^{* *}$ & $-.212^{*}$ & $-218^{*}$ \\
EC & $.474 * * *$ & .145 & 1.00 & $.495^{* * *}$ & $.480^{* * *}$ \\
CS & $.575^{* * *}$ & $-.968^{* * *}$ & -.139 & .016 & .009 \\
CU & $.711^{* * *}$ & .009 & $.552^{* * *}$ & $.709^{* * *}$ & $.915^{* * *}$ \\
EPP & $.214^{*}$ & $-.815^{* * *}$ & $-.369^{* * *}$ & $-.254 *$ & $-.330^{* *}$ \\
DL & $-.462 * * *$ & -.106 & $-.218^{*}$ & $-.607 * * *$ & $-.228^{*}$ \\
\hline
\end{tabular}

$C R S-F P V$ Coparenting Relationship Scale-Father's Prenatal Version, $L C S$ lack of coparenting support, $C C$ coparenting conflict, $C D$ coparenting disagreement, $C U$ coparenting undermining, $C R S$ Coparenting Relationship Scale, $C A$ coparenting agreement, $C C L$ coparenting closeness, $E C$ exposure to conflict, $E P P$ endorse partner parenting, $D L$ division of labor

$* p<.05 ; * * p<.01 ; * * * p<.001$

Table 5 Correlations between CRS-FPV subscales and depressive and anxious symptoms, adult attachment (avoidance and anxiety) and partner's relationship quality

\begin{tabular}{lllll}
\hline & LCS & CC & CD & CU \\
\hline Depressive symptoms & .001 & $.213^{*}$ & $.278^{* *}$ & .165 \\
Anxious symptoms & .177 & .166 & $.242^{*}$ & .145 \\
Adult attachment (avoidance) & $.310^{* *}$ & $.382^{* * *}$ & $.312^{* *}$ & $.317 * *$ \\
Adult attachment (anxiety) & .164 & .164 & $.404 * * *$ & $.251^{*}$ \\
Positive partner's relationship & -.021 & -.196 & $-.227 *$ & -.159 \\
Negative partner's relationship & .127 & .176 & $.269 *$ & .169 \\
\hline
\end{tabular}

$C R S-F P V$ Coparenting Relationship Scale-Father's Prenatal Version, $L C S$ lack of coparenting support, $C C$ coparenting conflict, $C D$ coparenting disagreement, $C U$ coparenting undermining

$* p<.05 ; * * p<.01 ; * * * p<.001$

of partner's parenting) become salient. In addition, this study was conducted with participants in a different cultural context than the original measure development work. Studies have found that gender and cultural background may affect coparenting mental representations and behaviors (e.g., Feldman et al. 2010; Lindsey et al. 2005; Schoppe-Sullivan et al. 2008). During pregnancy parents start to develop mental representations of themselves as coparents (e.g., Feinberg 2003; Van Egeren 2003) and, dimensions of coparenting not identified by the CRS-FPV could be salient only in the postpartum period.

The CRS-FPV subscales presented good internal consistency ( $\alpha$ 's ranged from .69 to .94). All the subscales presented Cronbach's alpha of over .70, the benchmark for good internal consistency, except the shortest of the subscales, the coparenting undermining subscale (four items) whose alpha was only slightly lower (e.g., Field 2005).

The CRS-FPV also revealed good criterion validity. For example, depressive and anxious symptoms were positively associated with the coparenting disagreement subscale and the depressive symptoms were also positively associated with the coparenting conflict subscale. Associations between the adult attachment and the coparenting subscales were found. Both dimensions of adult attachment (avoidance and anxiety) were positively associated with the coparenting disagreement and the coparenting undermining subscales. The avoidance dimension of adult attachment was positively associated with the lack of coparenting support and coparenting conflict subscales. Associations between the positive and negative dimensions of the partner's relationship quality and the father's reports on the coparenting disagreement subscale were found in the expected direction. We used these variables to assess the CRS-FPV criterion validity because previous studies have found significant associations between them and coparenting (e.g., Bronte-Tinkew et al. 2007, 2009, 2010; McHale et al. 2004; Talbot et al. 2009).

\section{Limitations}

We point out some methodological limitations in this study. The voluntary nature of the participation may have led to a selection bias. Those who agreed to participate may be those who feel more involved and satisfied with the pregnancy experience. The sample was composed only of primiparous fathers. The CRS-FPV is a self-report measure of coparenting only validated for fathers during pregnancy and we have not evaluated it in terms of assessing coparenting among mothers during this period. Future studies should include observational measures of coparenting (e.g., Altenburger et al. 2014; Favez et al. 2013) in order to compare the development of father's coparenting mental representations with coparenting interactions during pregnancy. 


\section{Implications for Practice and Research}

The CRS-FPV may be used during pregnancy in order to identify fathers and families at risk of an unsuccessful transition to parenthood (e.g., Feinberg and Kan 2008; Feinberg 2002; Solmeyer et al. 2013), future negative family processes and fathers' psychological maladjustment. This self-report measure allows a multidimensional assessment of coparenting during pregnancy. For coparenting researchers, the CRS-FPV can be used in future studies about coparenting during pregnancy. The identification of specific dimensions of coparenting provided by the CRSFPV enables the development of new research about prenatal coparenting, the processes involved, and its association with infant developmental outcomes, and parents and family adjustment. Future studies may also examine the psychometric characteristics of the CRS-FPV in a sample composed of expectant mothers.

\section{Conclusion}

The present study contributes to the development of prenatal coparenting literature by suggesting the CRS-FPV as a reliable multidimensional measure to assess coparenting in fathers during pregnancy.

Acknowledgements This study was conducted at Psychology Research Centre (UID/PSI/01662/2013), University of Minho, and supported by the Portuguese Foundation for Science and Technology and the Portuguese Ministry of Education and Science through national funds and co-financed by FEDER through COMPETE2020 under the PT2020 Partnership Agreement (POCI-01-0145-FEDER-007653). This study was also supported by FEDER Funds through the Programa Operacional Factores de Competitividade - COMPETE and by National Funds through FCT - Fundação para a Ciência e a Tecnologia under the project PTDC/SAU/SAP/116738/2010.

\section{References}

Altenburger, L. E., Schoppe-Sullivan, S. J., Lang, S. N., Bower, D. J., \& Kamp Dush, C. M. (2014). Associations between prenatal coparenting behavior and observed coparenting behavior at 9-months postpartum. Journal of Family Psychology, 28, 495. https://doi. org/10.1037/fam0000012.

Biaggio, A. M., Natalicio, L., \& Spielberger, C. D. (1976). The development and validation of an experimental Portuguese form of the State-Trait Anxiety Inventory. In C. D. Spielberger \& R. DiasGuerrero (Eds.), Cross-cultural research on anxiety (pp. 29-40). Washington, DC: Wiley.

Brennan, K. A., Clark, C. L., \& Shaver, P. (1998). Self-report measures of adult romantic attachment. In J. A. Simpson \& W. S. Rholes (Eds.), Attachment theory and close relationships (pp. 46-76). New York: Guilford.

Bronte-Tinkew, J., Horowitz, A., \& Carrano, J. (2010). Aggravation and stress in parenting: Associations with coparenting and father engagement among resident fathers. Journal of Family Issues, 31, 525-555. https://doi.org/10.1177/0192513X0934014 7.

Bronte-Tinkew, J., Moore, K. A., Matthews, G., \& Carrano, J. (2007). Symptoms of major depression in a sample of fathers of infants: Sociodemographic correlates and links to father involvement. Journal of Family Issues, 28, 61-99. https://doi. org/10.1177/0192513X06293609.

Bronte-Tinkew, J., Scott, M. E., Horowitz, A., \& Lilja, E. (2009). Pregnancy intentions during the transition to parenthood and links to coparenting for first-time fathers of infants. Parenting: Science and Practice, 9(1-2), 1-35. https://doi.org/10.1080/1529519080 2656729.

Cattell, R. B. (1966). The scree test for the number of factors. Multivariate Behavioral Research, 1, 245-276. https://doi.org/10.1207/ s15327906mbr0102_10.

Cox, J. L., Holden, J. M., \& Sagovsky, R. (1987). Detection of postnatal depression. Development of the 10-item Edinburgh Postnatal Depression Scale. The British Journal of Psychiatry, 150, 782-786. https://doi.org/10.1192/bjp.150.6.782.

Favez, N., Frascarolo, F., Scaiola, C., \& Corboz-Warnery, A. (2013). Prenatal representations of family in parents and coparental interactions as predictors of triadic interactions during infancy. Infant Mental Health Journal, 34, 25-36. https://doi.org/10.1002/ imhj.21372.

Feinberg, M. (2002). Coparenting and the transition to parenthood: A framework for prevention. Clinical Child \& Family Psychology Review, 5, 173-195. https://doi.org/10.1023/A:1019695015110.

Feinberg, M. (2003). The internal structure and ecological context of coparenting: A framework for research and intervention. Parenting: Science and Practice, 3, 85-132. https://doi.org/10.1207/ S15327922PAR0302_01.

Feinberg, M. E., Brown, L. D., \& Kan, M. L. (2012). A multi-domain self-report measure of coparenting. Parenting: Science and Practice, 12, 1-21. https://doi.org/10.1080/15295192.2012.638870.

Feinberg, M. E., \& Kan, M. L. (2008). Establishing Family Foundations: Intervention effects on coparenting, parent/infant wellbeing, and parent-child relations. Journal of Family Psychology, 22, 253-263. https://doi.org/10.1037/0893-3200.22.2.253.

Feldman, R., Masalha, S., \& Derdikman-Eiron, R. (2010). Conflict resolution in the parent-child, marital, and peer contexts and children's aggression in the peer group: A process-oriented cultural perspective. Developmental Psychology, 46, 310-325. https://doi. org/10.1037/a0018286.

Field, A. (2005). Discovering statistics using SPSS. London: SAGE.

Figueiredo, B., \& Conde, A. (2011). Anxiety and depression symptoms in women and men from early pregnancy to 3-months postpartum: Parity differences and effects. Journal of Affective Disorders, 132, 146-157. https://doi.org/10.1016/j.jad.2011.02.007.

Figueiredo, B., Field, T., Diego, M., Hernandez-Reif, M., Deeds, O., \& Ascenio, A. (2008). Partner relationships during the transition to parenthood. Journal of Reprodutive and Infant Psychology, 26, 99-107. https://doi.org/10.1080/02646830701873057.

Kline, R. B. (2005). Principles and practice of structural equation modeling (2nd ed.). New York: Guilford.

Lindsey, E. W., Caldera, Y., \& Colwell, M. (2005). Correlates of coparenting during infancy. Family Relations, 54, 346-359. https://doi. org/10.1111/j.1741-3729.2005.00322.x.

Matthey, S., Barnett, B., Kavanagh, D. J., \& Howie, P. (2001). Validation of the Edinburgh Postnatal Depression Scale for men, and comparison of item endorsement with their partners. Journal of Affective Disorders, 64, 175-184. https://doi.org/10.1016/S0165 -0327(00)00236-6.

McHale, J. P., Kazali, C., Rotman, T., Talbot, J., Carleton, M., \& Lieberson, R. (2004). The transition to coparenthood: Parents' prebirth expectations and early coparental adjustment at 3 months 
postpartum. Development and Psychopathology, 16, 711-733. https://doi.org/10.1017/S0954579404004742.

Paiva, C. A., \& Figueiredo, B. (2010). Study of validation of the Portuguese version of the inventory «Experiences in Close Relationships». Interpersona, 4, 237-270.

Parfitt, Y., \& Ayers, S. (2014). Transition to parenthood and mental health in first-time parents. Infant Mental Health Journal, 35, 263-273. https://doi.org/10.1002/imhj.21443.

Pedro, M. F., Ribeiro, T., \& Shelton, K. H. (2012). Marital satisfaction and partners' parenting practices: The mediating role of coparenting behavior. Journal of Family Psychology, 26, 509-522. https:// doi.org/10.1037/a0029121.

Schoppe-Sullivan, S. J., Altenburger, L. E., Settle, T. A., Dush, K., Sullivan, C. M., J. M., \& Bower, D. J. (2014). Expectant fathers' intuitive parenting: Associations with parent characteristics and postpartum positive engagement. Infant Mental Health Journal, 35, 409-421. https://doi.org/10.1002/imhj.21468.

Schoppe-Sullivan, S. J., Brown, G. L., Cannon, E. A., Mangelsdorf, S. C., \& Sokolowski, M. S. (2008). Maternal gatekeeping, coparenting quality, and fathering behavior in families with infants. Journal of Family Psychology, 22, 389-398. https://doi. org/10.1037/0893-3200.22.3.389.

Scrimgeour, M. B., Blandon, A. Y., Stifter, C. A., \& Buss, K. A. (2013). Cooperative coparenting moderates the association between parenting practices and children's prosocial behavior. Journal of Family Psychology, 27, 506-511. https://doi.org/10.1037/a0032 893.
Solmeyer, A., Feinberg, M., Coffman, D., \& Jones, D. (2013). The effects of the Family Foundations Prevention Program on coparenting and child adjustment: A mediation analysis. Prevention Science. https://doi.org/10.1007/s11121-013-0366-x.

Solmeyer, A. R., \& Feinberg, M. E. (2011). Mother and father adjustment during early parenthood: The roles of infant temperament and coparenting relationship quality. Infant Behavior and Development, 34, 504-514. https://doi.org/10.1016/j.infbe h.2011.07.006.

Spielberger, C. D., Gorsuch, R. L., Lushene, R., Vagg, P. R., \& Jacobs, G. A. (1983). Manual for the State-Trait Anxiety Inventory. STAI (Form Y). Palo Alto: Consulting Psychologists Press Inc.

Talbot, J. A., Baker, J. K., \& McHale, J. P. (2009). Sharing the love: Prebirth adult attachment status and coparenting adjustment during early infancy. Parenting: Science and Practice, 9, 56-77. https ://doi.org/10.1080/15295190802656760.

Van Egeren, L. A. (2003). Prebirth predictors of coparenting experiences in early infancy. Infant Mental Health Journal, 24, 278-295. https://doi.org/10.1002/imhj.10056.

Van Egeren, L. A. (2004). The development of coparenting over the transition to parenthood. Infant Mental Health Journal, 25, 453477. https://doi.org/10.1002/imhj.20019.

Van Egeren, L. A., \& Hawkins, D. P. (2004). Coming to terms with coparenting: Implications of definition and measurement. Journal of Adult Development, 11, 165-178. https://doi.org/10.1023/ B:JADE.0000035625.74672.0b. 